\title{
Biocompatible 3D printed magnetic micro needles
}

\author{
Mincho Kavaldzhiev ${ }^{1}$, Jose Efrain Perez ${ }^{2}$, Yurii Ivanov ${ }^{1}$, Andrea Bertoncini ${ }^{2}$, Carlo Liberale ${ }^{2}$ \\ and Jürgen Kosel $^{1}$ \\ 1: Computer, Electrical and Mathematical Science and Engineering \\ 2: Biological and Environmental Sciences and Engineering \\ e-mail : \\ Mincho.kavaldzhiev@kaust.edu.sa \\ Corresponding author e-mail : jurgen.kosel@kaust.edu.sa
}

\begin{abstract}
Biocompatible functional materials play a significant role in drug delivery, tissue engineering and single cell analysis. We utilized 3D printing to produce high aspect ratio polymer resist microneedles on a silicon substrate and functionalized them by iron coating. Two-photon polymerization lithography has been used for printing cylindrical, pyramidal, and conical needles from a drop cast IP-DIP resist. Experiments with cells were conducted with cylindrical microneedles with $630 \pm 15 \mathrm{~nm}$ in diameter with an aspect ratio of 1:10 and pitch of $12 \mu \mathrm{m}$. The needles have been arranged in square shaped arrays with various dimensions.

The iron coating of the needles was $120 \pm 15 \mathrm{~nm}$ thick and has isotropic magnetic behavior. The chemical composition and oxidation state were determined using energy electron loss spectroscopy, revealing a mixture of iron and $\mathrm{Fe} 3 \mathrm{O} 4$ clusters.

A biocompatibility assessment was performed through fluorescence microscopy using calcein/EthD-1 live/dead assay. The results show a very high biocompatibility of the iron coated needle arrays.

This study provides a strategy to obtain electromagnetically functional microneedles that benefit from the flexibility in terms of geometry and shape of $3 \mathrm{D}$ printing. Potential applications are in areas like tissue engineering, single cell analysis or drug delivery.
\end{abstract}

\section{Introduction}

Materials and devices capable of providing enhanced therapeutic effects to drugs or highly controllable delivery attract significant attention in the areas of microbiology, bioengineering and medicine. Spherical beads or nanowires have been utilized for remote drug delivery (1) (2-4), killing $(5,6)$ and identification (7) of cancer cells, hyperthermia applications (8) or capturing cells $(9)$. Microneedles (10) have been adopted for transdermal administration of drugs $(11,12)$, single cell manipulation and delivery (13-15), neurodegenerative disease treatments (16) or glucose measurement (17). In most cases, the fabrication of these structures requires a complex process of plasma enhanced etching and many lithography steps with limited flexibility regarding structural geometries on the same substrate (18). Cost effective technologies to print polymer pillars (19), like nanoimprinting, have been competing with traditional photo lithography manufacturing to provide a large printing volume at low cost and with a resolution from nano to micro scale. However, the costs of such lithography equipment vary between $\$ 0.5 \mathrm{M}-\$ 10 \mathrm{M}(20)$ and rely on costly masks with persistent challenges to print high aspect ratio structures (21). In recent years, additive manufacturing techniques such as 3D printing have become viable methods for fabricating structures of customized shapes with a high resolution, aspect ratio and small length scale at affordable price $(\$ 0.25 \mathrm{M}(22)$ or less (23-26). The two photon polymerization process has been used to create bioscaffolds for tissue regeneration (27)), whereas vaccine loaded microneedles (28-30) showed the potential of this technology for fast scaling and prototyping of drug-loaded devices. Biological entities are in sizes ranging from about $500 \mu \mathrm{m}$ (Amoeba proteus) to $130 \mathrm{~nm}$ (HIV) (31). Most of the 3D printed microneedles are on the scale of several hundreds of microns, making it problematic for them to internalize into smaller cell bodies like photoreceptor cells $(25 \mu \mathrm{m})$ or red blood cells $(8 \mu \mathrm{m})(31)$. Microneedles from carbon and silicon $(2,14,32-35)$ with dimensions of $100 \mathrm{~nm}$ in diameter and $6 \mu \mathrm{m}$ in length, have been employed for forced internalization $(33,36)$ or self internalization $(37,38)$, but 
their fabrication requires the use of sophisticated workflows and has limited customization capabilities.

Various studies have shown cell culture growth on top of hollow or solid needles $(32,38-41)$. It was found that cell type, pitch, needle diameter and height (42-45) influence the cell differentiation and other factors, highlighting the importance of the ability to control the needles' design parameters.

In this work, we propose the fabrication of tailored, biocompatible and functional needles made of a $3 \mathrm{D}$ printed polymer with an iron surface coating. We have printed cylindrical, conical and pyramidal microneedles (46), with various aspect ratios and base geometries. Besides the magnetic properties of the coating, the iron surface is covered by a thin oxide layer (47), which offers potential for biofunctionalization (48). Examples of this include the functionalization of nickel nanowires with antibodies for cell separation (49), as well as the visualization of nanowire internalization (50). All of the needles are organized as arrays of square-shaped patches constituting of 225 needles printed on a Si substrate. We have targeted colon cancer cells (HTC 116), which exhibit a mean diameter of 15.4 $\mu \mathrm{m}(51,52)$, using a substrate with cylindrical needles printed on it. These needles are $630 \pm 15 \mathrm{~nm}$ in diameter and $6 \mu \mathrm{m}$ in length with a pitch of $12 \mu \mathrm{m}$ to obtain approximately one needle per cell that is penetrating about half way into the cell's body.

\section{Methods}

\subsection{Microneedle fabrication}

Microneedles were prepared using a direct laser writing system (Nanoscribe, GmbH, Photo Professonal GT), utilizing two-photon polymerization, as illustrated in figure 1, on a silicon (Si) substrate and iron coated by sputtering deposition. Specifically, a single-side polished silicon (Si) (100) wafer was pre-cleaned with IPA and heated to $100^{\circ} \mathrm{C}$ to remove contaminants. Next, it was diced into $2.5 \times 2.5 \mathrm{~cm}^{2}$ pieces with a thickness of $525 \pm 0.5 \mu \mathrm{m}$ and a refractive index of 3.4401 (53) to be further used as a substrate. An IP-DIP (Nanoscribe) liquid resist was drop cast on the polished side of the Si die (figure 1(a)) and the printer objective lens (63x Zeiss microscope objective NA1.4) was directly dipped (Dip-In laser lithography (DiLL) configuration (54)) into the photoresist, serving as immersion and photosensitive medium at the same time. The needle shape was defined in 3D space by crosslinking the polymer by means of two photon polymerization process (55) starting with finding the surface of the Si substrate (figure 1(b)) followed by printing sequentially (figure 1(c)). This two photon absorption mechanism allows to polymerize the resist only in the focal spot, therefore allows $3 \mathrm{D}$ printing. In order to ensure adhesion of the printed structure to the substrate, the first laser voxel needs to partially lie inside of the substrate. This is controlled by the position of the stage offset, for which three examples are shown in figure 1(b). Our printing system relies on a high mismatch of the refractive indices of the resist and the substrate in order for the immersed objective lens to find the substrate surface. The difference in refractive indices between the IP-DIP resist and the Si wafer is 0.5 , which is much more than the required 0.05. The output laser power of the Photonic Professional GT system was adjusted to $15 \mathrm{~mW}$. 
The power of 3D printing lies in the possibility of tuning the specific needle shape. In order to obtain cylindrical needles a stage offset of 0.5 (figure 1(d)) has been used, which ensures a straight growth in the vertical direction, and the laser power was kept constant during the exposure, while stacking voxels in the vertical direction. Conical needles were obtained using a stage offset of 1 (figure 1(e)), which, compared to 0.5 avoids a straight growth in the vertical direction at the bottom. Voxels were then placed in circles on top of each other and the radius of the circles was linearly reduced until ending with a single voxel tip. Pyramidal needles were printed using the same dose, exposure time and stage offset as conical needles (figure 1(e)), starting either with a triangular base or square base, and continually decreasing the number of printed voxels per line, in order to have a sharp tip.

In all cases the piezo motor speed during printing was $42 \mu \mathrm{m} / \mathrm{s}$. After exposure, the sample was immersed in Mr-Dev 600 developer for $5 \mathrm{~min}$, after which a constant dose $0.05 \mathrm{ml}$ of IPA was added every 10 seconds for another 2 min (figure 1(f)).

Finally, the 3D printed needles were coated with Fe (MaTeck GmbH, 99.995\% purity, No.090805412), as shown in (figure 1(g)), by sputter deposition (902 PLC, Equipment Support Company LtD). The stage was rotated at $0.2 \mathrm{~m} / \mathrm{s}$ to achieve uniformity with a pressure of $6 \mathrm{e}^{-6} \mathrm{mTorr}$ at room temperature and the substrate was maintained at a $45^{\circ}$ angle for better sidewall coverage. The plasma conditions were $10 \mathrm{mTorr}, 30 \mathrm{sccm}$ Ar and $125 \mathrm{~W}$ power, resulting in a deposition rate of $7 \mathrm{~nm} / \mathrm{min}$. The film and needle SEM topography image analysis were carried out with ImageJ software.

a )

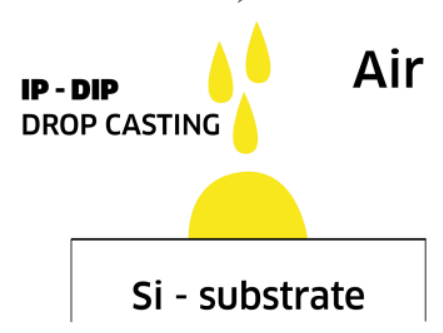

d)

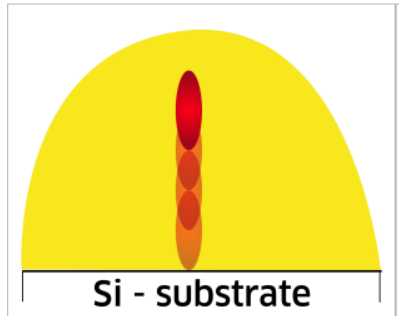

b )

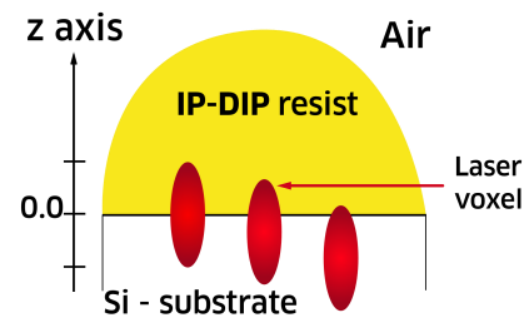

e )

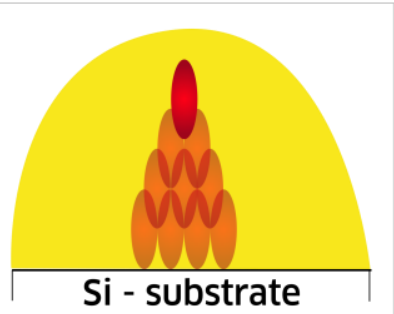

f)

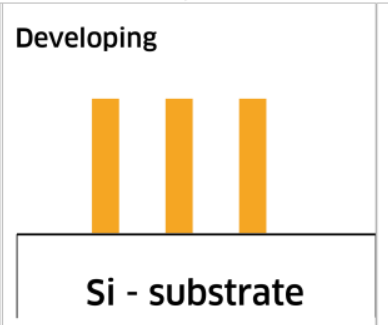

c)

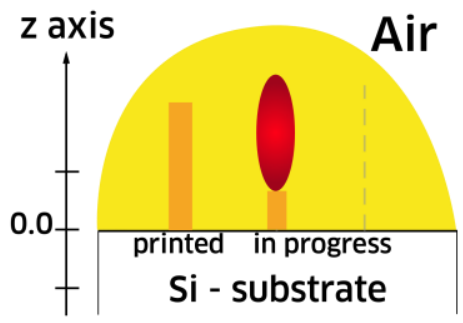

g )

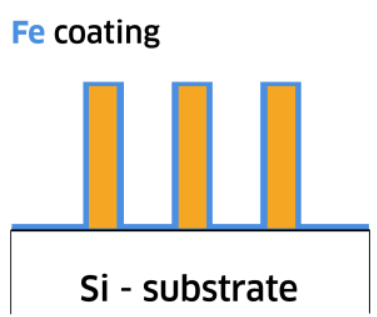

Figure 1: Process flow of microneedle fabrication. a) Drop casting of the IP-Dip resist on the Siwafer. b) Finding the substrate surface and three examples of stage offset through which the position of the substrate surface with respect to the first voxel is controlled. c) 3D printing perpendicular to the substrate (z-axis direction) with $15 \mathrm{~mW}$ of laser power. d) Printing of cylindrical structure by stacking of laser voxels. e) Printing of conical or pyramidal structures by 2D stacking of voxels with larger base (circular, triangular, square). f) Array of 3D printed needles after development. g) Fe sputtering to coat the printed structures.

\subsection{Cell culture}

HCT 116 colorectal carcinoma cells (ATCC ${ }^{\circledR}$ CCL-247 ${ }^{\mathrm{TM}}$ ) were cultured according to vendor indications. Briefly, McCoy's 5A modified medium (ATCC ${ }^{\circledR}$ ) was supplemented with $10 \%$ fetal bovine serum (Gibco $\left.{ }^{\circledR}\right)$ and L-glutamine and then used to culture the cells in a $37^{\circ} \mathrm{C}$ humidified incubator with 5\% CO2. Cells were then detached using Accutase (StemPro®) and counted using the standard trypan blue staining method. The microneedle substrate was first sterilized in ethanol for an hour, then thoroughly washed with phosphate buffered saline (PBS) and McCoy's medium before cell seeding. 


\subsection{Material Characterization}

The magnetic characterization of the Fe-coated needles was conducted using a Superconducting quantum interference device (Evercool SQUID-VSM system, Quantum Design). A $200 \mathrm{~nm}$ in diameter Omniprobe and focused ion beam have been used to fix the needle on a copper substrate (prod \# 460-2031-S) for the SQUID measurement. Transmission Electron Microscopy (TEM, FEI, TITAN) and Scanning Electron Microscopy (SEM, FEI, QUANTA 600) were used to characterize the geometry, morphology and composition of the microneedles as well as the magnetic coating.

\subsection{Confocal and SEM microscopy}

For imaging the cultured cells were washed with PBS and then fixed in $2.5 \%$ glutaraldehyde in $0.1 \mathrm{M}$ cacodylate buffer for $2 \mathrm{~h}$ at room temperature. After fixation, cells were washed with $0.1 \mathrm{M}$ cacodylate buffer three times for $15 \mathrm{~min}$ each wash. The cells were then post-fixed in $1 \%$ osmium tetroxide (Electron Microscopy Sciences, Hatfield, PA, USA) in cacodylate buffer for $1 \mathrm{~h}$ in the dark, after which the cells were washed with deionized water three times for 15 min each. This was followed by a serial dehydration using ethanol at $10 \%, 30 \%, 50 \%, 70 \%, 90 \%$ and $100 \%$ for 5 min each. The sample was then subjected to critical point drying in 100\% ethanol and then sputter-coated with $5 \mathrm{~nm}$ of gold-palladium before SEM imaging.

The cell viability of HCT 116 cells grown on the microneedle substrate was evaluated using the LIVE/DEAD Viability/Cytotoxicity Kit (Molecular Probes ${ }^{\mathrm{TM}}$ ). After incubation for 24 hours on the microneedles, cells were incubated with a $2 \mu \mathrm{M}$ calcein AM and $4 \mu \mathrm{L}$ EthD- 1 working solution for 40 minutes at room temperature. The cells were washed with PBS and then their interface with the microneedles was imaged using a Zeiss LSM 710 inverted confocal microscope. For staining the cell membrane with the green dye Cell Mask ${ }^{\mathrm{TM}}$ (Molecular Probes ${ }^{\mathrm{TM}}$ ), the cells were incubated with $1 \mathrm{x}$ CellMask ${ }^{\mathrm{TM}}$ working solution for 10 minutes at $37^{\circ} \mathrm{C}$, then thoroughly washed with PBS before imaging.

SEM microscopy was performed with cells cultured as previously mentioned and then seeded on the microneedles. After 24 hours of incubation, cells observed under a Quanta 3D SEM for a total time of imaging of 10 minutes.

\section{Results and discussions}

\subsection{Microneedles}

Figure 2(a) shows an SEM image of a single 3D printed cylindrical resist microneedle, which has a rounded tip with an average diameter of $580 \mathrm{~nm} \pm 20 \mathrm{~nm}$. A laser power of $15 \mathrm{~mW}$ and $100 \mathrm{~ms}$ exposure time provides excellent reproducibility for cylindrical needles up to $6 \mu \mathrm{m}$ in length. 
The wave pattern on the surface of the polymer needle is a result of the back reflection of the laser $(780 \mathrm{~nm})$ light from the Si substrate, forming an interference pattern with a pitch $(\lambda / 2 \mathrm{n}$, with $\mathrm{n}=1.52)$ of $256 \mathrm{~nm}$ in the polymerized area $(56,57)$. Figure 2(b) reveals that the surface morphology from the resist needle is transferred to the iron surface. An array of cylindrical, iron coated needles with pitch of $12 \mu \mathrm{m}$ is shown in figure 2(c). Using the same power $(15 \mathrm{~mW})$ and exposure dose $(100 \mathrm{~ms})$ as for cylindrical needles, we have successfully printed a variety of shapes shown in figure $2(\mathrm{~d})-(\mathrm{g})$, demonstrating the versatility of this method in terms of design.

a)

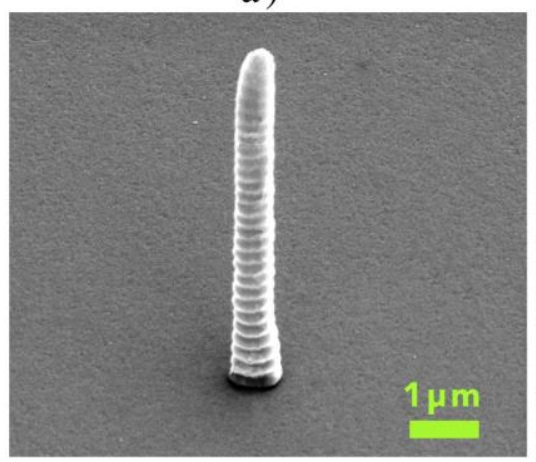

d)

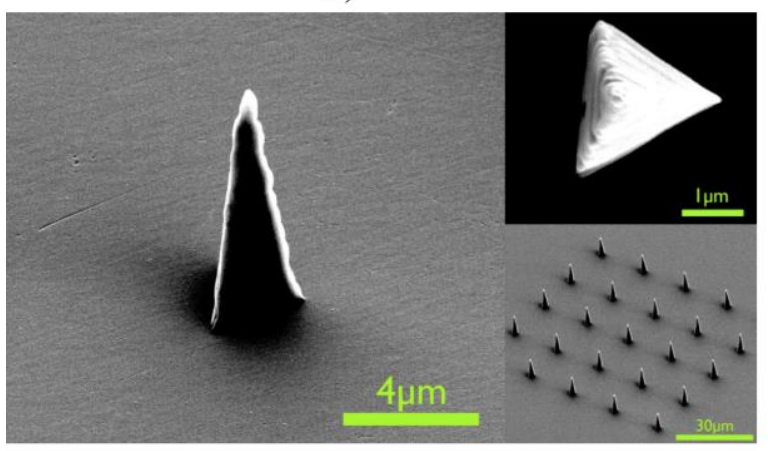

f)

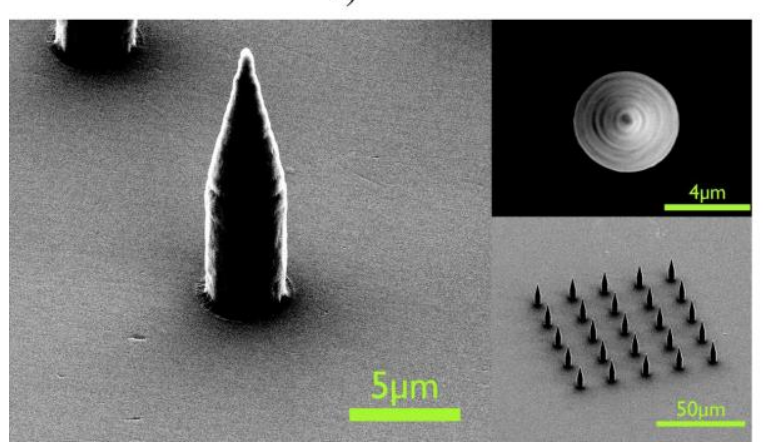

b)
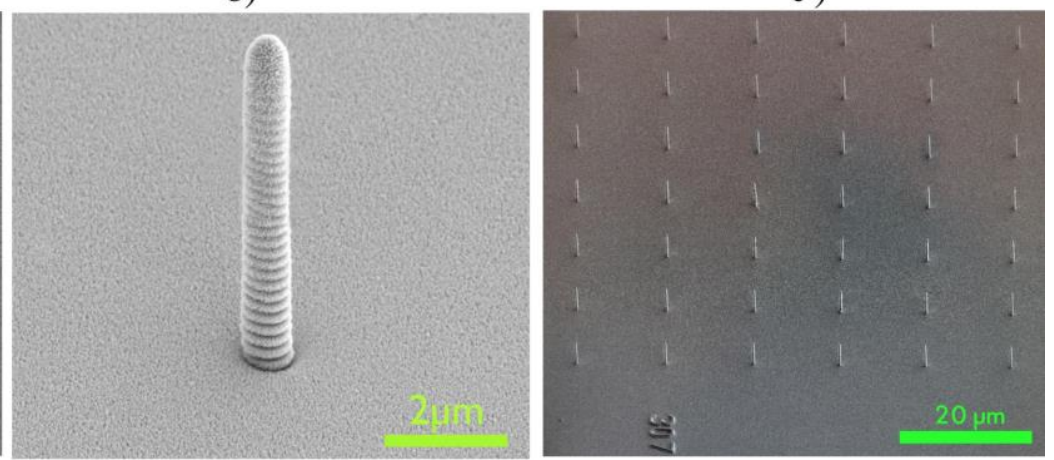

e)

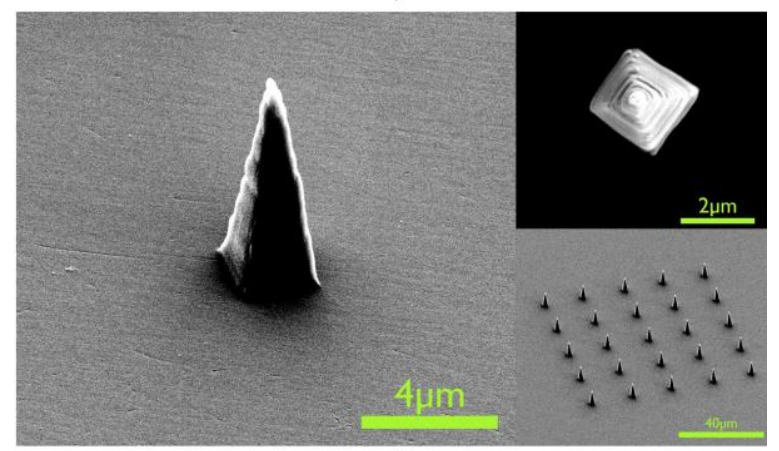

g)

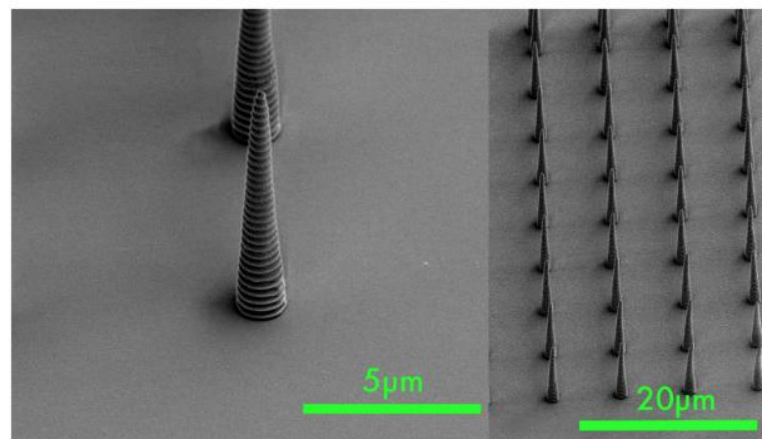

Figure 2: (a) SEM image of a 3D printed cylindrical polymer resist needle and (b) of a cylindrical needle coated with a $120 \mathrm{~nm}$ thick Fe layer. (c) SEM image of a patch of cylindrical needles after coating with $6 \mu \mathrm{m}$ length and $12 \mu \mathrm{m}$ pitch on a silicon substrate. SEM images of (d) 3D printed pyramids with triangular base, (e) square base, (f) cylinders with sharp tip and (g) conical needles with their respective arrays on a Si polished substrate. 
a)

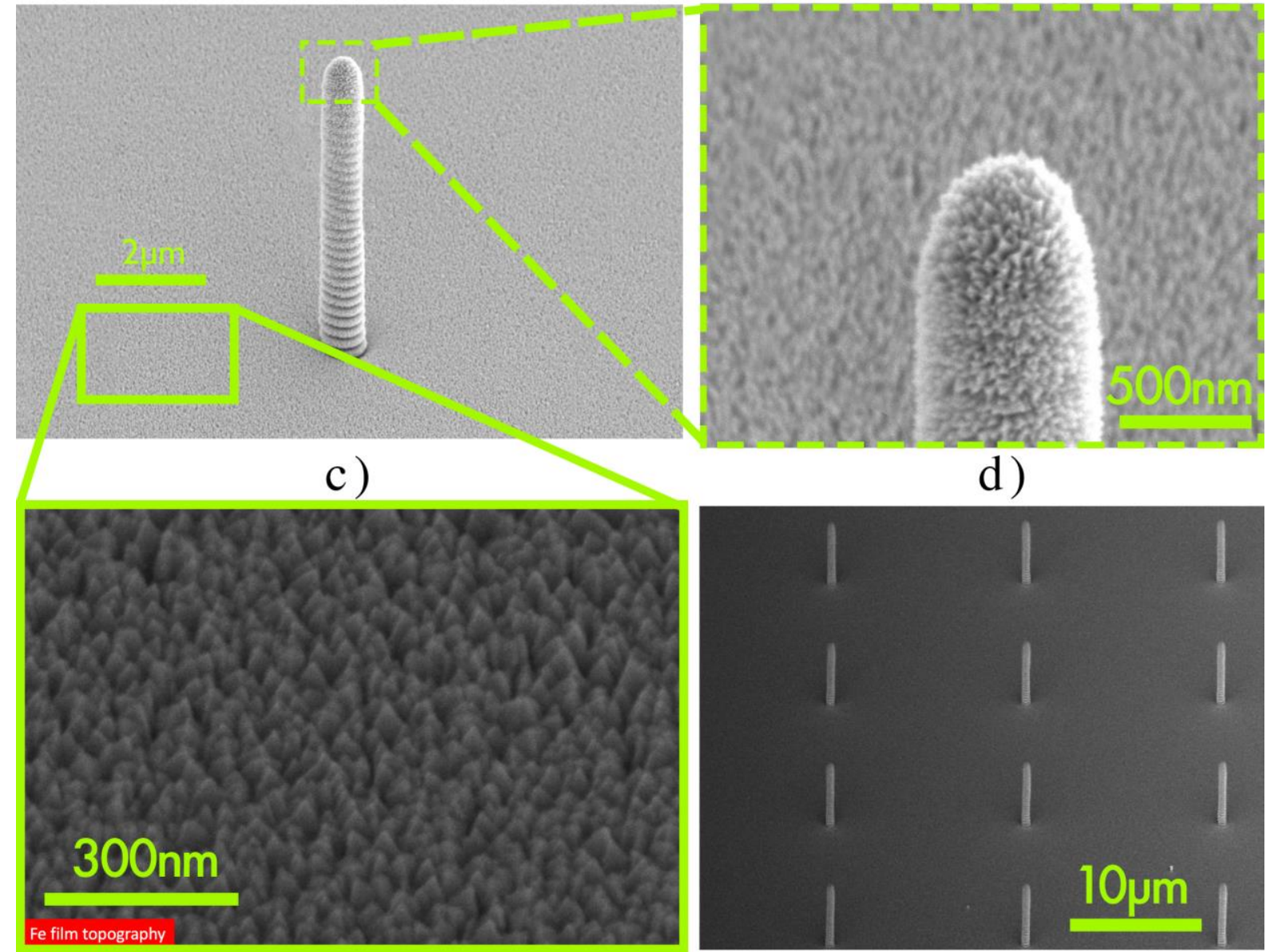

Figure 3 : SEM images of the sputtered iron film on the 3D printed resin needles (a), showing the roughness of the film on the needle tip (b) and of the film on the substrate (c). Example of a 3D printed microneedle array coated with an iron film by sputter deposition with $6 \mu \mathrm{m}$ long needles spaced by $12 \mu \mathrm{m}(\mathrm{d})$.

Closer views of the tip of an iron coated needle (figure 3(a)) and the film on the Si surface are shown in figure 3(b) and figure 3(c), revealing consistent coverage of the micro needle structures. The roughness of the film at the needle tip and the $\mathrm{Si}$ is $40 \pm 15 \mathrm{~nm}$ on average. There are no apparent deformations after deposition, even for the higher aspect ratio needles (figure 3(d)). Tomography analysis from TEM images of the sputtered iron shell in figure 4(a)-(c) reveals a homogeneous shell coating with a thickness of $120 \pm 15 \mathrm{~nm}$ (figure 4(c)). The polymer resist layer has been extracted by simply breaking the substrate with a blade, and the detached shells have been put into dimethyl sulfoxide solvent for polymer dissolution. The produced shells were imaged with TEM combination of dark and bright fields figure 4(a). 
a)

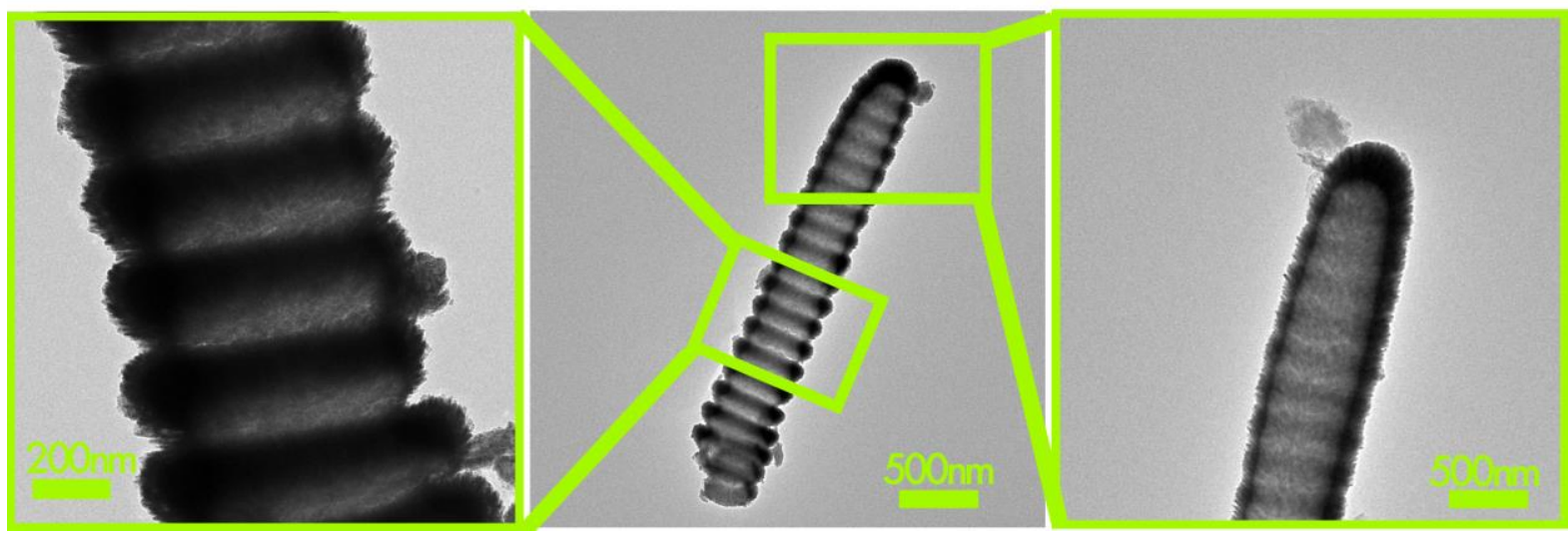

Figure 4 : TEM images of different parts of the iron coated needles. Middle section (a), entire needle (b) and tip (c), showing an Fe layer thickness of $120 \pm 15 \mathrm{~nm}$.

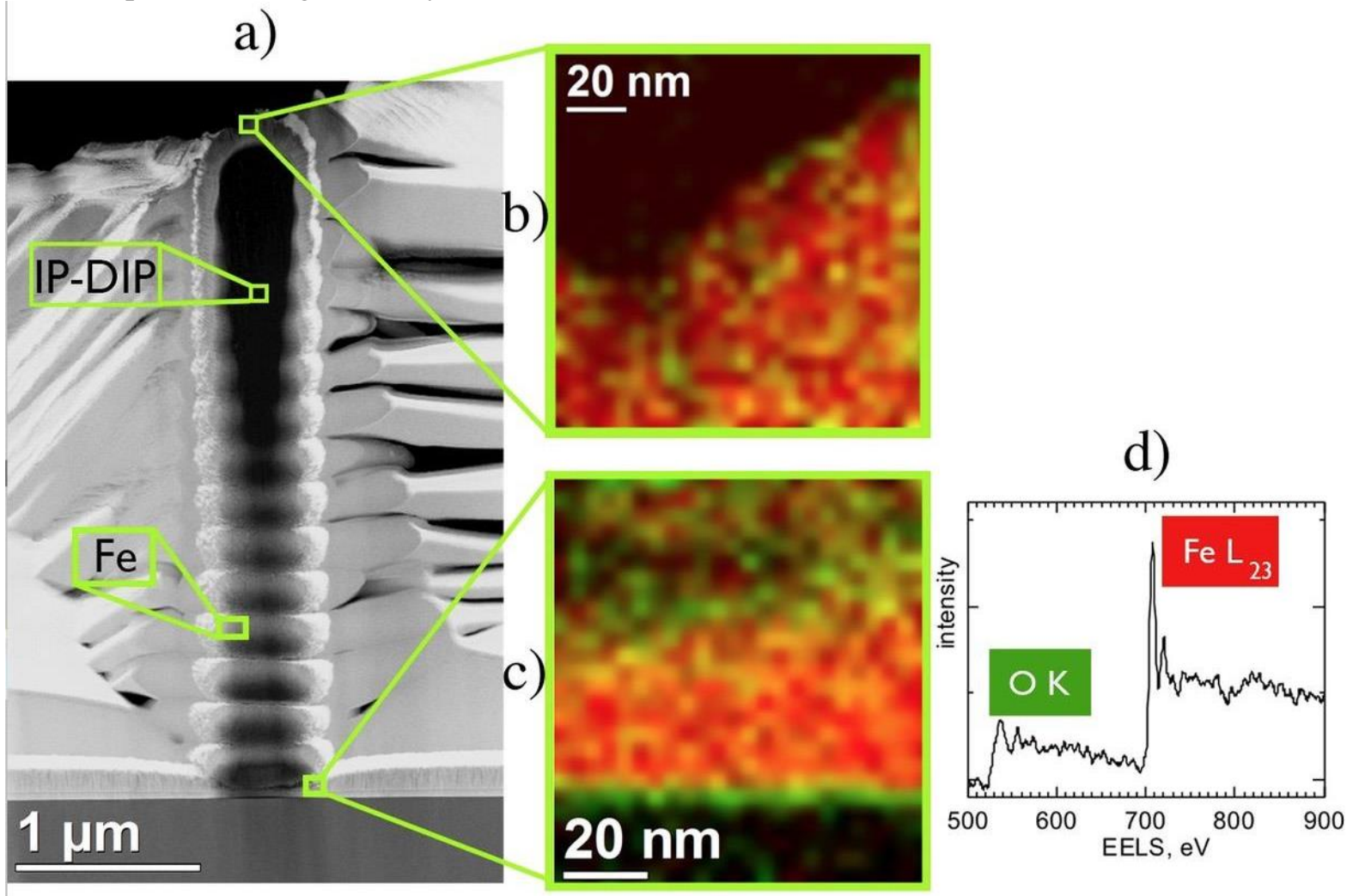

Figure 5: Cross section image (a) and Electron Energy Loss Spectroscopy (EELS) element mapping $(\mathrm{b}, \mathrm{c})$ of an $\mathrm{Fe}$ coated 3D printed microneedle (color coding represents $\mathrm{Fe}$ in red and $\mathrm{O}$ in green). The EELS spectroscopy reveals a mixture of $\mathrm{Fe}$ and $\mathrm{Fe}_{3} \mathrm{O}_{4}(\mathrm{~d})$.

The bright field TEM image in (figure 5(a)) shows a cross section of a 3D printed microneedle with Fe coating. EELS maps of the deposited films on the surface of the polymer needle and at its bottom are shown in figure 5(b) and figure 5(c), respectively. They reveal an $\mathrm{Fe} / \mathrm{O}$ atomic ratio of more than 1 , which corresponds to a mixture of iron and iron oxide. The analysis of the white lines ratio of $\mathrm{Fe}$ L2/L3 peaks (figure 5(c)) revealed the valence of the Fe to be close to the one for magnetite (58). This oxidation of the Fe film occurs naturally, when being exposed to air.

Magnetization curves of a square-shaped patch of cylindrical needles with $6 \mu \mathrm{m}$ height, $630 \pm 15 \mathrm{~nm}$ diameter, $12 \mu \mathrm{m}$ pitch and a total sample area of $3 \times 2 \mathrm{~mm}^{2}$ are shown in figure $6(\mathrm{a})$. The samples have been demagnetized before the measurements. In the parallel direction (in plane applied field) to 
the substrate, a softer magnetic behavior is found and a coercive field of $134 \mathrm{Oe}$, compared to the direction perpendicular (out of plane applied field) to the substrate (parallel direction to the needles) and a coercive field of 263 Oe. Hence, the magnetic properties are mainly determined by the Fe thin film on the substrate and its shape anisotropy, favoring an in-plane magnetization direction.

a)

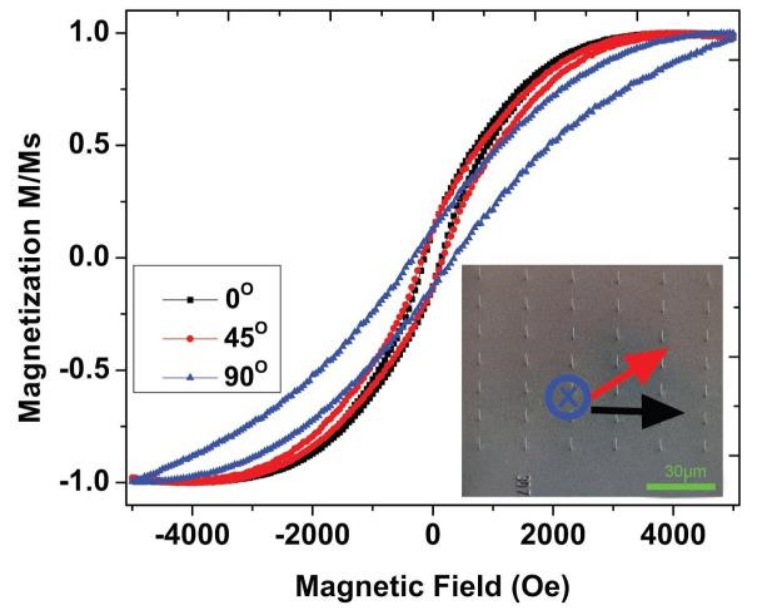

b)

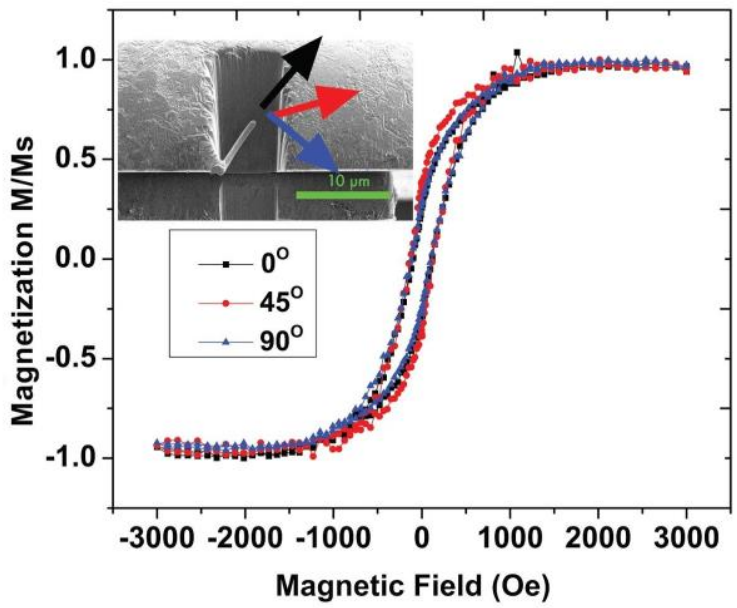

Figure 6: (a) Magnetization curves of 750 3D printed cylindrical needles of $6 \mu \mathrm{m}$ in height and a separation distance of $12 \mu \mathrm{m}$ coated with a $0.12 \mu \mathrm{m}$ thick Fe layer. The inset shows an array of such needles with markers for the directions of the applied field. (b) A single cylindrical $6 \mu \mathrm{m}$ long microneedle coated with a $0.12 \mu \mathrm{m}$ thick Fe layer. The inset shows the placement of the cylindrical needle inside the Omniprobe copper well. The arrows correspond to the directions of the applied magnetic field.

In order to obtain the magnetization curve of an individual Fe coated 3D printed needle, one needle was carefully removed from the substrate and placed on a standard Omniprobe copper lift off grid (prod \# 460-2031-S). The results obtained using a SQUID are illustrated in figure 6(b). The sample has been demagnetized before the measurements and the measurements are taken at $300 \mathrm{~K}$. The needle shows a coercive field of 143 Oe in both in and out of plane direction, which is comparable to iron oxide core shell nanowires when exposed to air oxidation not annealed (59). All three directions show isotropic magnetic behavior. This can be explained by the typical domain pattern in such tubes, which favors neither of the three directions. Rather, the domains are arranged circumferentially around the tubes (60).

\subsection{Biocompatiblity and microneedle-cell interface}

The cell viability results show an excellent biocompatibility (figure 7(a)). The non-fluorescent dye calcein AM is converted to its green fluorescent variant calcein by intracellular esterases, which are only active in live, viable cells. The red fluorescent dye EthD-1 is only permeant to a damaged cell membrane, as in the case of a dying cell. This indicates that interfacing the microneedles with HCT 116 cells does not compromise cell viability, and therefore is a viable substrate for intracellular delivery.

Confocal microscopy studies were carried out using the calcein AM and the cell membrane dye CellMask ${ }^{\mathrm{TM}}$ in order to closely observe the interface of the micro needles and the cells. An orthogonal section of a Z-stack of cells grown on the microneedles shows the cytosol-bound calcein (figure 7(b)). It can be observed that the cells wrap around the microneedles, which appear as black, nonfluorescent sections indented throughout the cells. To confirm this interaction, the same analysis was performed using the CellMask ${ }^{\mathrm{TM}}$ (figure $7(\mathrm{c})$ ). In this case, as the stained cell membrane folds around the microneedles (figure 7(e),(f)), these appear as bright green fluorescent sections. 

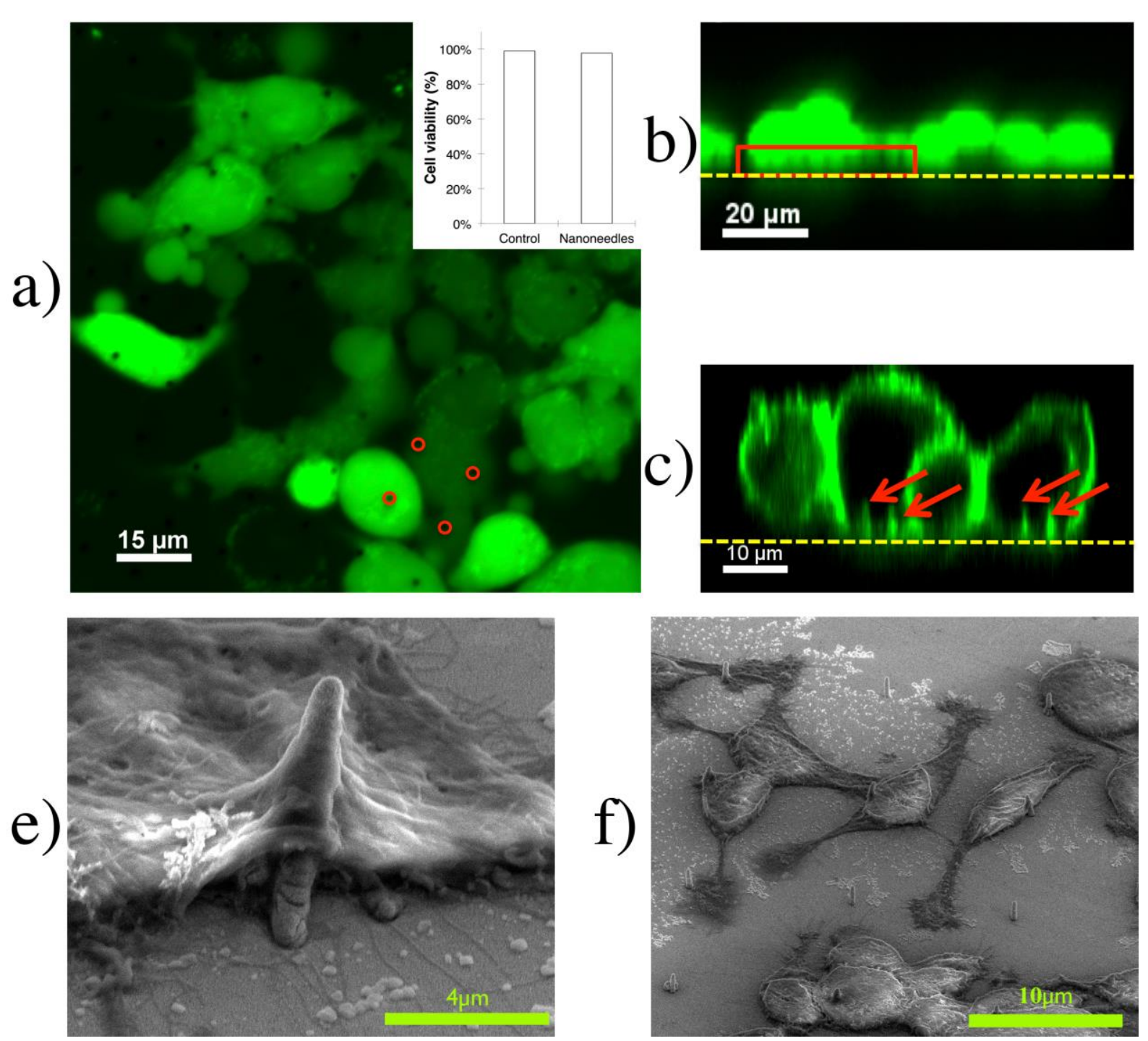

Figure 7: Confocal and SEM microscopy analysis of the interface of the microneedles and HCT 116 cells. (a) Cell viability of cells stained with calcein AM/EthD-1 under fluorescence microscope (top view). The black spots are micro needles (4 are highlighted by red circles as examples). (b) Confocal imaging cross-section view of a Z-stack of cells interfacing with the microneedles and stained with calcein AM. The microneedles appear as black, non-fluorescent sections (examples are marked by a red rectangle). (c) Cell membrane staining under the same imaging conditions, showing the stained membrane is folding around the microneedles, which appear as fluorescent sections (marked by red arrows). The yellow dashed line represents the position of the substrate. (e) SEM image of cell wrapping around a cylindrical microneedle on a Si substrate. (f) SEM image of an array of microneedles with cells seeded on top.

\section{Conclusions}

A fabrication process for 3D printed polymer micro needles coated with iron by sputter deposition has been developed. This process combines the flexibility of 3D printing in terms of shape and dimensions with the functional properties of a magnetic metal layer. Cylindrical, conical and pyramidal microneedles with aspect ratios of 1:10 have been fabricated and organized into square patches. Iron was chosen for its biocompatibility, and its TEM/EELS analysis shows a native oxide layer. It also revealed polycrystalline structures and the presence of $\mathrm{Fe}_{3} \mathrm{O}_{4}$ and $\mathrm{Fe}$, for which wellestablished coating techniques with biologically active materials like antibodies exist. In addition, its magnetic properties could be exploited in various ways like electromagnetic heating or nanoparticle manipulation. However, it should be straightforward to utilize other metals as coating layers for the 
needles, and the presented fabrication method is flexible in this regard, utilizing common sputter deposition. The deployment of such a platform with cylindrical micro needles on HTC116 cells proves that there are no effects on cell viability over a $24 \mathrm{~h}$ period with normal cell growth, confirming its potential as substrate for cell studies. Imaging studies of the cell-micro needle interface show that the cells adjust to the micro needles substrate by wrapping their membrane around the micro needles.

1. Gijs MAM. Magnetic bead handling on-chip: new opportunities for analytical applications. Microfluid Nanofluid. Springer-Verlag; 2004;1(1):22-40.

2. Shalek AK, Robinson JT, Karp ES, Lee JS, Ahn DR, Yoon MH, et al. Vertical silicon nanowires as a universal platform for delivering biomolecules into living cells. Proc Natl Acad Sci USA [Internet]. 2010 Feb 2;107(5):1870-5. Available from: http://www.pnas.org/cgi/doi/10.1073/pnas.0909350107

3. Zaher A, Li S, Wolf KT, Pirmoradi FN, Yassine O, Lin L, et al. Osmotically driven drug delivery through remote-controlled magnetic nanocomposite membranes. Biomicrofluidics. AIP Publishing; 2015 Sep;9(5):054113.

4. Yi Y, Zaher A, Yassine O, Kosel J, Foulds IG. A remotely operated drug delivery system with an electrolytic pump and a thermo-responsive valve. Biomicrofluidics. AIP Publishing; 2015 Sep;9(5):052608.

5. Contreras M, Sougrat R, Zaher A, Ravasi T, Kosel J. Non-chemotoxic induction of cancer cell death using magnetic nanowires. IJN. Dove Press; 2015 Mar;Volume 10:2141-13.

6. Martínez-Banderas AI, Aires A, Teran FJ, Perez JE, Cadenas JF, Alsharif N, et al. Functionalized Magnetic Nanowires for Chemical and Magneto-mechanical Induction of Cancer Cell Death. Sci Rep.

7. Cancer Cell Identification by Using ZnO Nanowires Modified by Carboxyalkylphosphonic Acids. 2014.

8. Alonso J, Khurshid H, Sankar V, Nemati Z, Phan MH, Garayo E, et al. FeCo nanowires with enhanced heating powers and controllable dimensions for magnetic hyperthermia. J Appl Phys. 2015 May 7;117(17):17D113-5.

9. Li J, Qi C, Lian Z, Han Q, Wang X, Cai S, et al. Cell-Capture and Release Platform Based on Peptide-Aptamer-Modified Nanowires. ACS Appl Mater Interfaces. American Chemical Society; 2016 Feb 3;8(4):2511-6.

10. Kim Y-C, Park J-H, Prausnitz MR. Microneedles for drug and vaccine delivery. Advanced Drug Delivery Reviews. Elsevier B.V; 2012 Nov 1;64(14):1547-68.

11. Fernando GJP, Chen X, Primiero CA, Yukiko SR, Fairmaid EJ, Corbett HJ, et al. Nanopatch targeted delivery of both antigen and adjuvant to skin synergistically drives enhanced antibody responses. J Control Release. 2012 Apr 29;159(2):215-21.

12. Lee H, Choi TK, Lee YB, Cho HR, Ghaffari R, Wang L, et al. A graphene-based electrochemical device with thermoresponsive microneedles for diabetes monitoring and therapy. Nature Nanotech. Nature Publishing Group; 2016 Mar 21.

13. Chen L, Liu X, Su B, Li J, Jiang L, Han D, et al. Aptamer-Mediated Efficient Capture and Release of T Lymphocytes on Nanostructured Surfaces. Adv Mater. WILEY-VCH Verlag; 
2011 Oct 11;23(38):4376-80.

14. Han S-W, Nakamura C, Miyake J, Chang S-M, Adachi T. Single-Cell Manipulation and DNA Delivery Technology Using Atomic Force Microscopy and Nanoneedle. J Nanosci

Nanotechnol. American Scientific Publishers; 2014 Jan;14(1):57-70.

15. Hanson L, Zhao W, Lou H-Y, Lin ZC, Lee SW, Chowdary P, et al. Vertical nanopillars for in situ probing of nuclear mechanics in adherent cells. Nature Nanotech. Nature Research; 2015 May 31;10(6):554-62.

16. Hood RL, Andriani RTJ, Emch S, Robertson JL, Rylander CG, Rossmeisl JHJ. Fiberoptic Microneedle Device Facilitates Volumetric Infusate Dispersion During Convection-Enhanced Delivery in the Brain. Lasers Surg Med. 2013 Sep;45(7):418-26.

17. Yu J, Zhang Y, Ye Y, DiSanto R, Sun W, Ranson D, et al. Microneedle-array patches loaded with hypoxia-sensitive vesicles provide fast glucose-responsive insulin delivery. Proc Natl Acad Sci USA. National Acad Sciences; 2015 Jul 7;112(27):8260-5.

18. He B, Yang Y, Yuen MF, Chen XF, Lee CS, Zhang WJ. Vertical nanostructure arrays by plasma etching for applications in biology, energy, and electronics. Nano Today. Elsevier Ltd; 2013 Jun 1;8(3):265-89.

19. Viela F, Granados D, Ayuso-Sacido A, Rodríguez I. Biomechanical Cell Regulation by High Aspect Ratio Nanoimprinted Pillars. Adv Funct Mater. 2016 Jun 20;26(31):5599-609.

20. Nanotechnologies S. About Nanoimprint. 2016.

21. Scheer H-C, Mayer A, Dhima K, Wang S, Steinberg C. Challenges with high aspect ratio nanoimprint. Microsyst Technol. Springer Berlin Heidelberg; 2013 Nov 23;20(10-11):1891-8.

22. GT P. Nanoscribe Photonic Professional GT review - 3D printer. 2016.

23. Holmes B, Bulusu K, Plesniak M, Zhang LG. A synergistic approach to the design, fabrication and evaluation of 3D printed micro and nano featured scaffolds for vascularized bone tissue repair. Nanotechnology. IOP Publishing; 2016;27(6).

24. Nano 3D printing hits the fast track. Physics Today. 2012 n/a;65:80-.

25. Freymann von G, Ledermann A, Thiel M, Staude I, Essig S, Busch K, et al. Three-

Dimensional Nanostructures for Photonics. Adv Funct Mater. WILEY-VCH Verlag; 2010 Apr 9;20(7):1038-52.

26. Park SY, Choi J-W, Park J-K, Song EH, Park SA, Kim YS, et al. Tissue-engineered artificial oesophagus patch using three-dimensionally printed polycaprolactone with mesenchymal stem cells: a preliminary report. Interact Cardiovasc Thorac Surg. Oxford University Press; 2016 Jun;22(6):712-7.

27. Pan J-F, Li S, Guo C-A, Xu D-L, Zhang F, Yan Z-Q, et al. Evaluation of synovium-derived mesenchymal stem cells and 3D printed nanocomposite scaffolds for tissue engineering. Science and Technology of Advanced Materials. IOP Publishing; 2015 Aug;16(4).

28. Ovsianikov A, Chichkov B, Mente P, Monteiro-Riviere NA, Doraiswamy A, Narayan RJ. Two Photon Polymerization of Polymer?Ceramic Hybrid Materials for Transdermal Drug Delivery. International Journal of Applied Ceramic Technology. Blackwell Publishing Inc; 2007 Jan;4(1):22-9. 
29. Lu Y, Mantha SN, Crowder DC, Chinchilla S, Shah KN, Yun YH, et al.

Microstereolithography and characterization of poly(propylene fumarate)-based drug-loaded microneedle arrays. Biofabrication. IOP Publishing; 2015 Sep 25;7(4):1-13.

30. Ali Z, Türeyen EB, Karpat Y, Çakmakc1 M. Fabrication of Polymer Micro Needles for Transdermal Drug Delivery System Using DLP Based Projection Stereo-lithography. Procedia CIRP. 2016;42:87-90.

31. Goodsell DS. The Machinery of Life. New York, NY: Springer Science \& Business Media; 2013. $1 \mathrm{p}$.

32. VanDersarl JJ, Xu AM, Melosh NA. Nanostraws for Direct Fluidic Intracellular Access. Nano Lett [Internet]. 2012 Aug 8;12(8):3881-6. Available from:

http://pubs.acs.org/doi/abs/10.1021/n1204051v

33. Obataya I, Nakamura C, Han S, Nakamura N, Miyake J. Nanoscale operation of a living cell using an atomic force microscope with a nanoneedle. Nano Lett. American Chemical Society; 2005 Jan;5(1):27-30.

34. Yan S, Xu Y, Yang J, Wang H, Jin Z, Wang Y. A novel fabrication method of silicon nanoneedles using MEMS TMAH etching techniques. Nanotechnology. IOP Publishing; 2011 Mar 25;22(12):125301.

35. Chen X, Kis A, Zettl A, Bertozzi CR. A cell nanoinjector based on carbon nanotubes. Proc Natl Acad Sci USA. National Acad Sciences; 2007 May 15;104(20):8218-22.

36. Yum K, Yu M-F, Wang N, Xiang YK. Biofunctionalized nanoneedles for the direct and siteselective delivery of probes into living cells. Biochimica et Biophysica Acta (BBA) - General Subjects. 2011 Mar;1810(3):330-8.

37. Peer E, Artzy-Schnirman A, Gepstein L, Sivan U. Hollow Nanoneedle Array and Its Utilization for Repeated Administration of Biomolecules to the Same Cells. ACS Nano. 2012 Jun 26;6(6):4940-6.

38. Chiappini C, De Rosa E, Martinez JO, Liu X, Steele J, Stevens MM, et al. Biodegradable silicon nanoneedles delivering nucleic acids intracellularly induce localized in vivo neovascularization. Nature Materials [Internet]. Nature Publishing Group; 2015 May 1;14(5):532-9. Available from: http://www.nature.com/doifinder/10.1038/nmat4249

39. Zhu X, Kwok SY, Yuen MF, Yan L, Chen W, Yang Y, et al. Dense diamond nanoneedle arrays for enhanced intracellular delivery of drug molecules to cell lines. Journal of Materials Science. Springer US; 2015 Aug 19;50(23):7800-7.

40. McKnight TE, Melechko AV, Hensley DK, Mann DGJ, Griffin GD, Simpson ML. Tracking Gene Expression after DNA Delivery Using Spatially Indexed Nanofiber Arrays. Nano Lett. 2004 Jul;4(7):1213-9.

41. Prinz CN. Interactions between semiconductor nanowires and living cells. Journal of Physics: Condensed Matter. IOP Publishing; 2015 May 25;27(23):1-11.

42. Piret G, Perez M-T, Prinz CN. Neurite outgrowth and synaptophysin expression of postnatal CNS neurons on $\mathrm{GaP}$ nanowire arrays in long-term retinal cell culture. Biomaterials. 2013 Jan;34(4):875-87.

43. Bonde S, Berthing T, Madsen MH, Andersen TK, Buch-Månson N, Guo L, et al. Tuning InAs 
Nanowire Density for HEK293 Cell Viability, Adhesion, and Morphology: Perspectives for Nanowire-Based Biosensors. ACS Appl Mater Interfaces. American Chemical Society; 2013 Nov 13;5(21):10510-9.

44. Kuo S-W, Lin H-I, Ho JH-C, Shih Y-RV, Chen H-F, Yen T-J, et al. Regulation of the fate of human mesenchymal stem cells by mechanical and stereo-topographical cues provided by silicon nanowires. Biomaterials. $2012 \mathrm{Jul} ; 33(20): 5013-22$.

45. Piret G, Perez M-T, Prinz CN. Substrate porosity induces phenotypic alterations in retinal cells cultured on silicon nanowires. RSC Advances. The Royal Society of Chemistry; 2014;4(53):27888-97.

46. Rovere A, Toma A, Prato M, Bertoncini A, Cerea A, Piccoli R, et al. Conical nanoantenna arrays for terahertz light. IEEE; 2016. pp. 1-1.

47. Song M-M, Song W-J, Bi H, Wang J, Wu W-L, Sun J, et al. Cytotoxicity and cellular uptake of iron nanowires. Biomaterials. 2010 Mar;31(7):1509-17.

48. Tanase M, Bauer LA, Hultgren A, Silevitch DM, Sun L, Reich DH, et al. Magnetic Alignment of Fluorescent Nanowires. Nano Lett. American Chemical Society; 2001 Mar;1(3):155-8.

49. Gao N, Wang H, Yang E-H. An experimental study on ferromagnetic nickel nanowires functionalized with antibodies for cell separation. Nanotechnology. 2010;21(10).

50. Fung AO, Kapadia V, Pierstorff E, Ho D. Induction of cell death by magnetic actuation of nickel nanowires internalized by fibroblasts. The Journal of Physical .... 2008.

51. Tahara M, Inoue T, Miyakura Y, Horie H, Yasuda Y, Fujii H, et al. Cell diameter measurements obtained with a handheld cell counter could be used as a surrogate marker of G2/M arrest and apoptosis in colon cancer cell lines exposed to SN-38. Biochem Biophys Res Commun. 2013 May 17;434(4):753-9.

52. Qiu X, De Jesus J, Pennell M, Troiani M, Haun JB. Microfluidic device for mechanical dissociation of cancer cell aggregates into single cells. Lab Chip. The Royal Society of Chemistry; 2015;15(1):339-50.

53. Chandler-Horowitz D, Amirtharaj PM. High-accuracy, midinfrared (450 $\mathrm{cm}[\sup -1] \leq \omega \leq 4000$ $\mathrm{cm}[\mathrm{sup}-1]$ ) refractive index values of silicon. J Appl Phys. AIP Publishing; 2005;97(12):123526.

54. Bueckmann T, Stenger N, Kadic M, Kaschke J, Froelich A, Kennerknecht T, et al. Tailored 3D Mechanical Metamaterials Made by Dip-in Direct-Laser-Writing Optical Lithography. Adv Mater. WILEY-VCH Verlag; 2012;24(20):2710-4.

55. Lay CL, Lee YH, Lee MR, Phang IY, Ling XY. Formulating an Ideal Protein Photoresist for Fabricating Dynamic Microstructures with High Aspect Ratios and Uniform Responsiveness. ACS Appl Mater Interfaces. American Chemical Society; 2016 Mar 30;8(12):8145-53.

56. Madou MJ. Fundamentals of Microfabrication. CRC Press; 2002. 1 p.

57. Mack CA. Analytical expression for the standing wave intensity in photoresist. Appl Opt. Optical Society of America; 1986 Jun 15;25(12):1958-61.

58. Ivanov YP, Alfadhel A, Alnassar M, Perez JE, Vazquez M, Chuvilin A, et al. Tunable magnetic nanowires for biomedical and harsh environment applications. Sci Rep. 2016;6. 
59. Ju Hun Lee, Jun Hua Wu, Ji Sung Lee, Ki Seok Jeon, Hae Ryong Kim, Jong Heun Lee, et al. Synthesis and Characterization of Fe-FeO2 Core-Shell Nanowires. IEEE Transactions on Magnetics. 2016 Aug 31;44(11):3950-3.

60. Kammouni El R, Kurlyandskaya GV, Vázquez M, Volchkov SO. Magnetic properties and magnetoimpedance of short $\mathrm{CuBe} / \mathrm{CoFeNi}$ electroplated microtubes. Sensors \& Actuators: A Physical. Elsevier B.V; 2016 Sep 1;248:155-61. 